\title{
Methicillin-resistant Staphylococcus aureus infected pseudo-arthrosis of the distal tibia treated with debridement, mesh cage, autologous grafting and locking plate fixation
}

\author{
Silas N. S. Motsitsi
}

Received: 18 February 2008/ Accepted: 29 August 2008/Published online: 9 September 2008

(C) Springer-Verlag 2008

\begin{abstract}
Infected non-union of long bones is a challenge to manage. It has a high morbidity and mortality. Treatment is very demanding and has a significant complication rate. Methicillin-resistant infected non-union has a higher morbidity and mortality compared to Methicillin-sensitive Staphylococcus aureus infection. Approximately half of all Staphylococci isolated in infected orthopaedic operations are caused by Methicillin-resistant S. aureus. We present a 42-year-old patient who had Methicillin-resistant S. aureus infected pseudo-arthrosis of the tibia that was treated with debridement, mesh cage, autologous bone-grafting, and plating of the tibia. The outcome was satisfactory despite residual limb deformity and discrepancy.
\end{abstract}

Keywords Methicillin-resistant Staphylococcus aureus . Infected non-union $\cdot$ Mesh cage $\cdot$ Locking plate

\section{Background}

The majority of long bone fractures will unite when managed in accordance with current orthopaedic practices. Approximately less than $1 \%$ will develop non-union. Nonunion is a formidable challenge to treat [1].

Infected non-union has a significant morbidity and mortality: infected non-union of long bones in B host carries a $90 \%$ rate of limb loss.

In infections following orthopaedic surgery, isolated Staphylococci are reported to be Methicillin-resistant

S. N. S. Motsitsi ( $)$

Department of Orthopaedic Surgery, Kalafong Hospital, University of Pretoria, Private Bag x 396, Pretoria, South Africa

e-mail: silas.motsitsi@up.ac.za
(MRSA) in up to 50\% of cases [2]. Unfortunately, there is no well-established protocol for the management of this serious infection. We report a case of MRSA-infected pseudo-arthrosis of the distal tibia treated with local debridement, cylindrical mesh cage, autologous grafting, and stabilization of the tibia with a locking plate.

\section{Case presentation}

A 42-year-old male patient was involved in a car accident. He sustained grade III B open fracture of the right distal tibia and fibula. The posterior tibialis artery was irreparably damaged. The wound was debrided and the fracture stabilized with an external fixator. He subsequently underwent two additional surgical interventions to effect union. The procedures were unsuccessful. He developed infection. Culture results identified resistant Methicillin-resistant Staphylococcus aureus MRSA as the causative organism. It was sensitive to vancomycin, rifampicin, and cotrimoxazole (sulfamethoxazole-trimethoprim). Infection was suppressed and the leg was immobilized in a Plaster of Paris.

He presented himself to our Trauma Clinic 6 months after the injury. His main complaints were; limb deformity, pain, shortening, and occasional draining sinus.

On local examination he had antero-lateral deformity of the right leg, scarring, swelling, signs of inflammation, but no actively draining sinus. There was no abscess. There was a weak dorsalis pedis pulse, no palpable posterior tibialis pulse, and the neurological status was clinically intact. He had $7.0 \mathrm{~cm}$ of limb length discrepancy. Clinically he had infected non-union of the right distal tibia.

Relevant blood investigations showed; $\mathrm{c}$ reactive protein $(\mathrm{CRP})=22 \mathrm{mg} / \mathrm{l}$ (normal range 0-8), and erythrocyte 
sedimentation rate $(\mathrm{ESR})=18 \mathrm{~mm} / \mathrm{h}$ (normal range 1-30).

Plain radiographs of the leg showed a $2.0 \mathrm{~cm}$ of tibial defect, sclerotic bone ends, no sequestrum, and plated distal fibula. The screws were loose (Fig. 1). No any other investigations were deemed necessary.

Oral antibiotics were stopped for 6 weeks. Multiple bone biopsies were taken from the fracture site after stopping oral antibiotics for the said period. Cultures for fungi and bacteria (aerobic and anaerobic) were negative. After discussions about different reconstructive procedures, their pros and cons, the patient preferred a one-stage definitive surgical procedure.

A one-stage surgical strategy was adopted: local debridement of the dead bone and soft tissue, removal of implant, repeat of microscopy, culture and sensitivity, filling of tibial defect with a mesh cage packed with $2.0 \mathrm{~g}$ of vancomycin-impregnated autologous bone-graft and stabilization of the tibia with locking plate (Fig. 2). The approach was lateral. The plate was placed laterally. No tibial or fibular shortening was necessary. The skin was closed primarily. The procedure was uneventful.

Methicillin-resistant Staphylococcus aureus was cultured from specimens taken intra-operatively. The organism was sensitive to the afore-mentioned antibiotics. Intravenous vancomycin was administered for 7 days. Oral rifampicin and cotrimoxazole were administered for

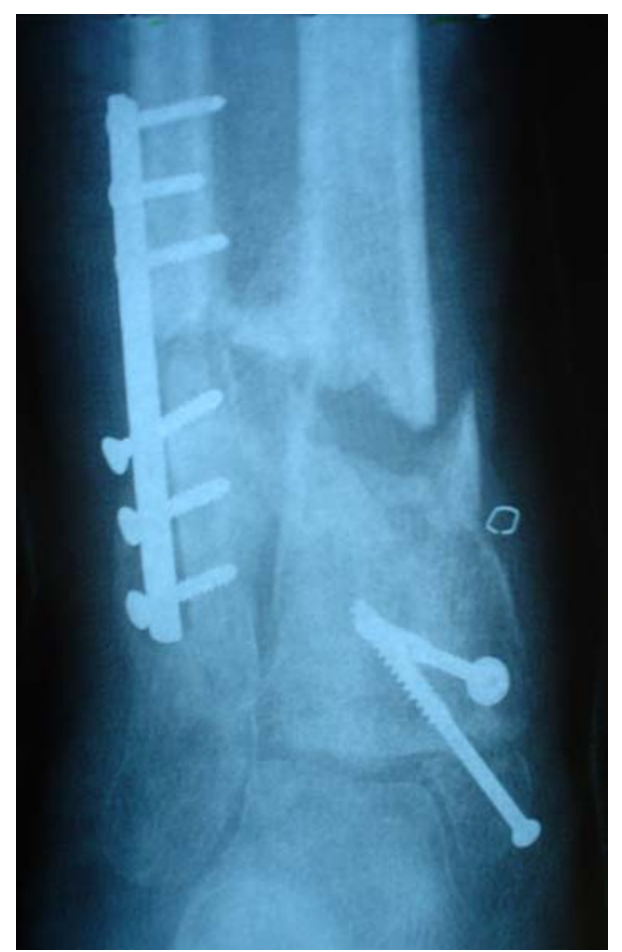

Fig. 1 Pre-operative radiograph shows tibial defect, sclerotic ends of the tibia and failing fibular implant

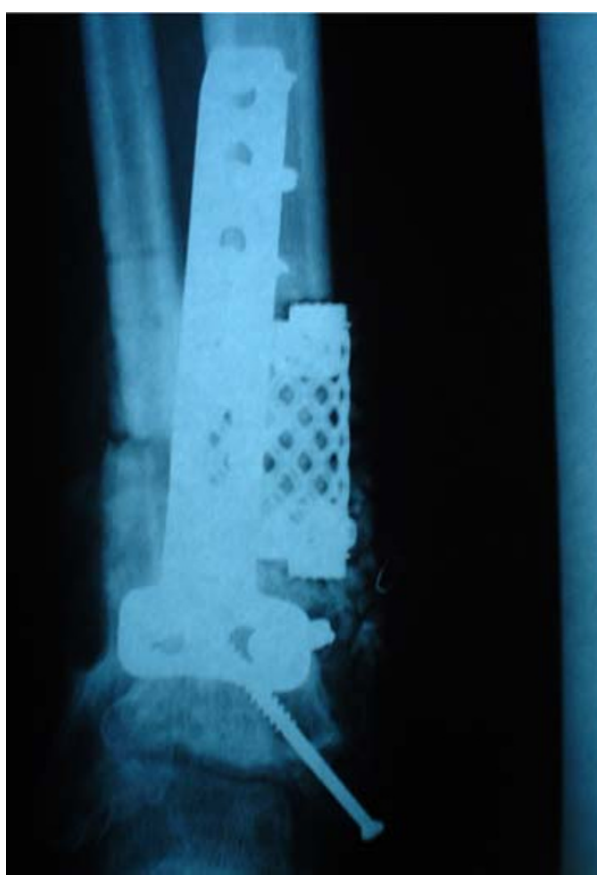

Fig. 2 The defect in the tibia is managed with titanium cage packed with autologous bone graft. The construct is stabilized with a locking plate

6 weeks. The post-operative course was uneventful. He was instructed to be non-weight bearing for the first 6 weeks, followed by partial-weight bearing (10\% of his weight) for the next 6 weeks and full-weight bearing thereafter. He resumed his professional duties 6 months after the operation.

Follow-up radiographs of the leg were done at 6 months intervals. No implant complications occurred.

He was reviewed for the last time 18 months after the operation. He had no complaints. He was full-weight bearing without crutches. He had a slight short-limb gait despite a shoe-raise. The leg showed antero-medial angulation, healed surgical scars, no signs of inflammation and mild stiffness of the ankle. There was a $4.0 \mathrm{~cm}$ limb length discrepancy. Radiographs showed solid union of the tibia and no implant complications (Fig. 3). The patient was satisfied with the outcome and he has not decided yet whether he will like the limb length discrepancy to be addressed surgically or not.

\section{Discussion}

Methicillin-resistant Staphylococcus aureus infected pseudo-arthrosis can be effectively managed by this less extensive procedure. The technique is simple and appealing. Limb length discrepancy can be corrected to a certain extent without compromising the limb's circulation. 


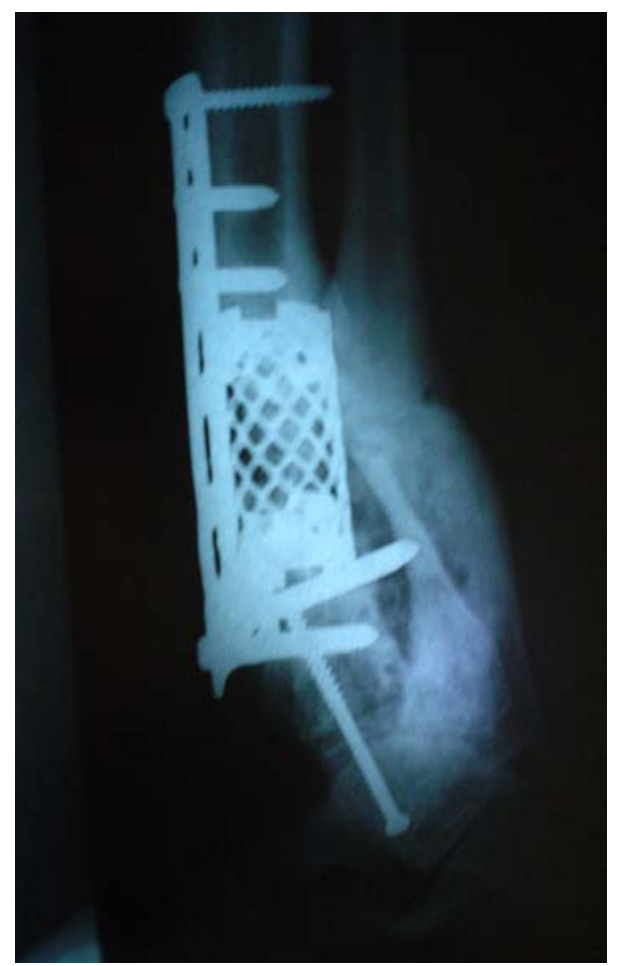

Fig. 3 The X-rays show bony union of the tibia. There is bone formation inside the cage. There is no implant complication. There is malunion of the tibia

Titanium cylindrical cages are extensively used in spinal surgery. Their use is well established and very successful. However, the use of cylindrical cages is occasionally used in limb trauma. They have been in use for over a decade. To the best of this author's knowledge, the first use of titanium mesh cages in limb trauma was presented as a poster in OTA meeting in America in 1995. Subsequently, other authors have published their experience with this technique in the English literature [2-6]. These publications are all case reports. There is no case of MRSA infected pseudo-arthrosis treated with this technique has been reported in the English literature. The use of titanium mesh cage in trauma is appealing because of its success rate, the ability of the construct to accommodate immediate limb mobility and rapid return to full weight bearing, the biologic advantage of cancellous bone grafting that can span a larger defect, and the major inherent limitations of other treatment alternatives [4]. Titanium has a unique feature of undergoing osteointegration [3].

Methicillin-resistant Staphylococcus aureus infection is costly to treat, has a high mortality and morbidity, and results in longer hospitalization compared to Methicillinsusceptible Staphylococcus infection [7]. Vancomycin is the first line agent for most cases of MRSA [8]. It has a poor tissue penetration. It reaches avascular areas by diffusion. It can reach very high concentration when delivered locally without causing systemic toxic effects [9, 10]. It is not effective against phagocytosed MRSA [8].

Rifampicin, when administered alone, tends to lead to rapid development of resistance by MRSA. The resistance can develop within 24-48 h [8]. The use of rifampicin with cotrimoxazole is a better regime for long-term use. The recommended long-term combination for MRSA is the combination of three drugs mentioned [8]. Another promising drug for long-term use in this infection is linezolid [3].

The limitation of this case is that the follow-up is relatively short. Implant-related infection can occur months or years later following fracture fixation.

Acknowledgments Written consent was obtained from the patient for publication of study.

Competing interests The author declares that he has no competing interests.

\section{References}

1. Cobos JA, Lindsey RW, Gugala Z (2000) The cylindrical titanium cage for treatment of a long bone segmental defect: description of a new technique and report of two cases. J Orthop Trauma 14(1):54-59

2. Harwood PJ, Talbot C, Dimoutsos M, Sunderland G, Shaw D, Wilcox MH, Giannoudis PV (2006) Early experience with linezolid for infections in orthopaedics. Injury 37:818-826

3. Shilov BL (2006) The first case of primary metacarpal V restoration with titanium mesh and cancellous bone graft. J Plastic Reconstr Aesthet Surg 59:1391-1393

4. Attias N, Lindsey RW (2006) Management of large segmental tibial defect using a cylindrical mesh cage. Clin Orthop Relat Res 450:259-266

5. Attias N, Lehman RE, Bodell LS, Lindsey RW (2005) Surgical management of a long segmental defect of the humerus using a cylindrical titanium mesh cage and plates. J Orthop Trauma 19(3):211-216

6. Panchbhavi VK, Trevino SG (2003) Use of titanium cages with cancellous bone graft in hindfoot fusion: literature and case report with complications. Foot Ankle Surg 9:51-55

7. Cosgrove SE, Qi Y, Kaye KS, Harbarth S, Karchmer AW, Carmeli Y (2005) The impact of Methicillin-resistant in Staphylococcus aureus bacteremia on patient outcome: mortality, length of stay, and hospital charges. Infect Control Hosp Epidemiol 26(2):166-174

8. Yamaoka T (2007) The bactericidal effects of anti-MRSA agents with rifampicin and sulfamethoxazole-trimethoprim against intracellular phagocytized MRSA. J Infect Chemother 13:141-146

9. Kinik H, Karaduman M (2008) Cierny-Mader Type III Chronic osteomyelitis: the results of patients treated with debridement, irrigation, vancomycin beads and systemic antibiotics. Int Orthop 32:551-558. doi: 10.1007/s00264-007-0342-9

10. Ozaki T, Yoshitaka T, Kunisada T, Dan'ura T, Naito N, Inoue H (1998) Vancomycin-Impregnated polymethylmethacrylate beads for methicilin-resitant Staphylococcus aureus (MRSA) infection: report of two cases. J Orthop Sci 3:163-168 